\section{Type 1 Diabetes Mellitus Following Acute Myo- cardial Infarction in a Young Adult}

Key words: type 1 diabetes mellitus, acute myocardial infarction, secondary prevention, microangiopathy, macroangiopathy

Cardiovascular disease is a chronic complication in diabetes mellitus with a significant attendant mortality and morbidity, and it is important for the patient to prevent the progression of macroangiopathy. However, the progression of macroangiopathy is not specified for glycemic control, which is different from microangiopathy such as diabetic retinopathy and nephropathy. We report the clinical course of a type 1 diabetes patient with a previous history of acute myocardial infarction in a young adult, which is useful for the discussion regarding the importance of glycemic control in the secondary prevention of cardiovascular events.

A 35-year-old man was admitted to our hospital suffering from chest pain on December 7, 1988. The electrocardiogram showed ST segment elevation in leads $\mathrm{V}_{1-5}$, and he was diagnosed with acute myocardial infarction (AMI) in the anterior septum area. Coronary angiography showed a total occlusion of the proximal left anterior descending artery, and balloon angioplasty of that lesion was performed. His body mass index was $19.6 \mathrm{~kg} / \mathrm{m}^{2}$. He had smoked about 40 cigarettes a day from the age of 18 . There was neither a family history of diabetes mellitus, nor cardiovascular disease. His past history was uneventful. There were no findings of dyslipidemia or hyperglycemia on laboratory data (total cholesterol, high density lipoprotein cholesterol, triglyceride, and fasting plasma glucose level were $195 \mathrm{mg} / \mathrm{dl}, 52 \mathrm{mg} / \mathrm{dl}$, $48 \mathrm{mg} / \mathrm{dl}$, and $99 \mathrm{mg} / \mathrm{dl}$, respectively). On follow-up coronary angiography, restenosis was not apparent, and he was treated with warfarin. Although he had chest pain once in the third month after the onset of AMI, he has had no recurrence of angina attack since then.

He was diagnosed with diabetes mellitus by an oral glucose tolerance test in July 1989 (fasting plasma glucose level, $127 \mathrm{mg} / \mathrm{dl}$; plasma glucose level in 2 hours, 321 $\mathrm{mg} / \mathrm{dl}$ ). His levels of plasma glucose and glycosylated hemoglobin A1c (HbA1c) had increased gradually since 1991 (Fig. 1). The islet cell antibody (ICA) was negative, the urinary excretion of C-peptide reactivity (U-CPR) was 65.3 $\mu \mathrm{g} / \mathrm{day}$, and there was no significant finding on abdominal ultrasonography, and thus he was diagnosed with type 2 diabetes then. He was treated with glibenclamide because his glycemic control did not improve with diet therapy alone. After 1992, he did not visit our hospital regularly, and it became impossible to measure his plasma glucose and thrombotest levels regularly. His drug compliance of glibenclamide was poor, and his warfarin treatment was changed to aspirin, too. In October 1994, he was admitted to our hospital suffering from thirst and general malaise. His fasting plasma glucose level was $335 \mathrm{mg} / \mathrm{dl}$, and the ketone body of urine was negative on admission. The level of $\mathrm{HbA1c}$ was $16.8 \%$, the U-CPR was $6.3 \mu \mathrm{g} /$ day, the ICA was negative, and he was treated with insulin. He had already had diabetic neuropathy, but not diabetic retinopathy and nephropathy.

He continued both multiple insulin injections and aspirin from 1994. In 1998, he was admitted to our hospital for diabetic ketoacidosis after having stopped insulin therapy on his own self-judgment (arterial blood gas analysis showed $\mathrm{pH}$ 6.998 , plasma glucose level was $673 \mathrm{mg} / \mathrm{dl}$, the ketone body of urine was positive). Anti-glutamic acid decarboxylase antibody (GAD-Ab) was $13.4 \mathrm{U} / \mathrm{ml}$, and he was finally diagnosed as a slow-onset patient with type 1 diabetes. His glycemic control worsened, and his microangiopathy such as diabetic retinopathy and nephropathy progressed gradually.

On March 2002, the intima-media thickness of his common carotid arteries (CCA) and internal carotid arteries (ICA) were $0.94 \mathrm{~mm}$ (left CCA), $0.82 \mathrm{~mm}$ (right CCA), 0.95 $\mathrm{mm}$ (left ICA), and $0.82 \mathrm{~mm}$ (right ICA), respectively. The B-mode ultrasonography of the carotid arteries did not show an atheromatous plaque. There was no significant finding on brain MRI, and artery stenosis in magnetic resonance angiography (MRA) of the brain was not apparent on January 2004. He has had neither chest pain nor changes in electrocardiogram and echocardiogram findings for 15 years.

Young adult patients with AMI are rare, and the rate of AMI in males is triple that in females. The risk factors of young adult patients with AMI are hyperlipidemia, cigarette smoking and familial history. There is a report that hypertension and diabetes mellitus are less important risk factors for AMI in young adults than those in older adults (1), although the cumulative mortality rate due to coronary artery disease in type 1 diabetic patients is far higher than the corresponding rate for non-diabetic patients (2). In addition, although the absolute risk for cardiac events is lower in the intensive treatment group for type 1 diabetes than in the conventional treatment group, the difference is not statistically significant (3). Therefore, the importance of glycemic control in the secondary prevention of AMI in young adults remains unclear.

In the present case, the risk factors of AMI in young adult were being male and a smoker. There was no history of obesity, dyslipidemia and hypertension. There were neither abnormal findings of physical examination nor repeating infarctions after AMI, and it was difficult to consider congenital amino acid metabolic disorders such as homocystinurea. Resulting from his clinical course for 15 years, poor glycemic control for type 1 diabetes mellitus following 


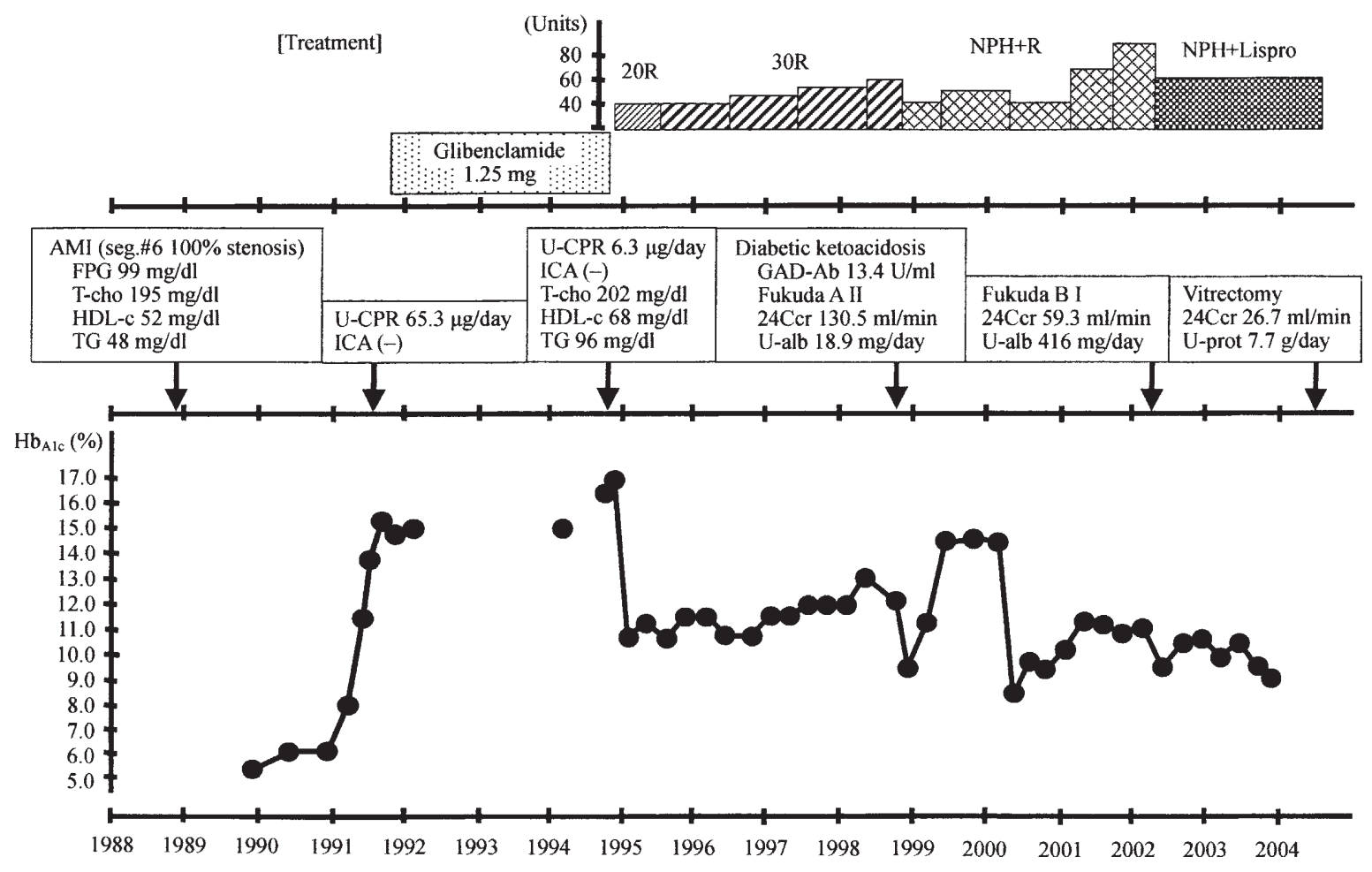

Figure 1. Clinical course from the onset of acute myocardial infarction (AMI) in a 35-year-old man. Type 1 diabetes was diagnosed after the onset of AMI, and diabetic complications such as retinopathy and nephropathy were progressed due to poor glycemic control throughout the course, but not re-infarction. FPG: fasting plasma glucose, T-cho: total cholesterol, HDL-c: high density lipoprotein cholesterol, TG: triglyceride, U-CPR: urinary excretion of C-peptide reactivity, ICA: islet cell antibody, GAD-Ab: anti-glutamic acid decarboxylase antibody, $24 \mathrm{Ccr}$ : creatinine clearance in 24 hours, U-alb: urinary excretion of albumin, U-prot: urinary excretion of protein. Clinical stage of diabetic retinopathy revealed by the severity scales of "Fukuda", "A II" means moderate non-proliferative diabetic retinopathy and "B I" means severe non-proliferative diabetic retinopathy (International clinical diabetic retinopathy disease severity scale).

AMI as a young adult was not associated with the coronary reinfarction, but with the progression of diabetic microangiopathy. Persistent hyperglycemia in young adults may not be a strong risk factor for coronary artery disease.

Carotid intima-media thickness is a well-established index of atherosclerosis that correlates with prevalent and incident coronary heart disease; there is a report that intensive insulin therapy for type 1 diabetes decreases progression of carotid intima-media thickness (4). This present case did not have obvious atherosclerotic change on B-mode ultrasonography of the carotid arteries and on brain MRA, although his glycemic control worsened. It is unclear whether the mechanism of the progression of macroangiopathy is the same as that of microangiopathy. Because type 2 diabetic patients often have multiple risk factors, the accumulation of type 1 diabetic patients with a history of AMI will be necessary to clarify whether purely hyperglycemia is a risk factor for macroangiopathy, which remains controversial at present.

Toshio KaHARA, Yoshiki NagATA, Hiroshi AKAHORI and Rika USUDA
From the Department of Internal Medicine, Toyama Prefectural Central Hospital, Toyama

Received for publication January 6, 2005; Accepted for publication March 23, 2005

Reprint requests should be addressed to Dr. Toshio Kahara, the Department of Internal Medicine, Toyama Prefectural Central Hospital, 2-2-78 Nishinagae, Toyama 930-8550

\section{References}

1) Hoit BD, Gilpin EA, Henning $\mathrm{H}$, et al. Myocardial infarction in young patients: an analysis by age subsets. Circulation 74: 712-721, 1986.

2) Krolewski AS, Kosinski EJ, Warram JH, et al. Magnitude and determinants of coronary artery disease in juvenile-onset, insulin-dependent diabetes mellitus. Am J Cardiol 59: 750-755, 1987.

3) The Diabetes Control and Complications Trial Research Group. Effect of intensive diabetes management on macrovascular events and risk factors in the Diabetes Control and Complications Trial. Am J Cardiol 75: 894-903, 1995.

4) Nathan DM, Lachin J, Cleary P, et al.; Diabetes Control and Complications Trial; Epidemiology of Diabetes Interventions and Complications Research Group. Intensive diabetes therapy and carotid intimamedia thickness in type 1 diabetes mellitus. N Engl J Med 348: 22942303, 2003. 